BULLETIN OF THE

AMERICAN MATHEMATICAL SOCIETY

Volume 80, Number 6, November 1974

\title{
CONDITIONAL INDEPENDENCE
}

\author{
BY ANATOLE BECK ${ }^{1}$ \\ Communicated May 28, 1974
}

1. Introduction. One of the basic concepts which pervades the whole of probability theory is that of independence. This is the condition which serves as part of the hypothesis of most of the major theorems of probability, most notably the laws of large numbers. In many cases, this condition is weakened to stationarity or uncorrelatedness, and usually there is a corresponding weakening in the sharpness of results. We shall define some new conditions which are weakenings of independence, and which yield many of the usual theorems in real or complex random variables, but which have noticeably weaker effect when applied to Banach space-valued random variables.

The study of probability in Banach spaces enables us to distinguish between the effects of probabilistic assumptions and those which flow from the geometry of the spaces in which the values are taken. In the strong law of large numbers, the extremes are the strong law of E. Mourier (1953) which holds in all Banach spaces, and the Kolmogorov strong law, which holds in Hilbert spaces and some others (these theorems are quoted below).

The new conditions defined here are properly weaker than independence, though some of them are sufficient to give the Kolmogorov strong law of large numbers in Hilbert spaces. The difference for Banach space-valued random variables, however, is striking.

2. Basic notions. We define random variables in Banach spaces by a natural extension of their definitions in the real case (cf., e.g., Beck [1], [2], [3]). In the same way, we define expectation, independence, stationarity, distribution, and symmetric random variable, in a natural way, and define, for each random variable with an expectation, the variance as $\operatorname{Var}(X)=\sigma^{2}(X)=$ $E\left(\|X-E(X)\|^{2}\right)$. As a generalization of orthogonality or uncorrelatedness, we define two random $\mathfrak{X}$-variables as weakly orthogonal if

$$
E\left(x^{*}(X-E(X)) x^{*}(Y-E(Y))\right)=0, \quad \forall x^{*} \in \mathfrak{X}^{*} .
$$

AMS (MOS) subject classifications (1970). Primary 60B05, 46B05, 46G10.

${ }^{1}$ This research was supported by the National Science Foundation. 
A collection of random $\mathfrak{X}$-variables is called mutually symmetric if each of them, $X$, is symmetrically distributed around 0 on each set $S$ taken from the field $F_{X}$ of sets in the probability space defined by the other random variables, i.e. for each such $X$ and $S, \chi_{S} X$ is symmetric around 0 . A collection is called conditionally independent if each of them has the property that its conditional expectation with respect to the field generated by the others is a constant.

A Banach space is called $B$-convex if there exist an integer $k \geqslant 2$ and an $\epsilon>0$ such that for every $k$-tuple $x_{1}, \cdots, x_{k}$ from the unit ball, we can assign + and - signs so that $\left\|x_{1} \pm x_{2} \pm \cdots \pm x_{k}\right\| \leqslant k(1-\epsilon)$. (Cf. Beck [2], [3].)

3. Known results.

1. THEOREM. Let $\left\{X_{i}\right\}$ be a sequence of real or complex random variables which are identically distributed, have expectation 0 , and are independent. Then this sequence obeys the strong law of large numbers (SLLN), i.e.,

$$
\frac{1}{n} \sum_{i=1}^{n} X_{i}(\omega) \rightarrow 0 \text { almost surely. }
$$

2. TheOREM (E. Mourier, 1953). Theorem 1 holds for random variables in any Banach space.

3. Theorem (Kolmogorov Strong law). Let $\left\{X_{i}\right\}$ be a sequence of independent random variables with $E\left(X_{i}\right)=0, \forall i$, and $\operatorname{Var}\left(X_{i}\right)$ satisfying $\sum_{i=1}^{n} \operatorname{Var}\left(X_{i}\right) / i^{2}<\infty$. Then the sequence $\left\{X_{i}\right\}$ satisfies the SLLN.

4. TheOREM. The Kolmogorov strong law holds in any Hilbert space.

5. THEOREM (BECK, 1961). Let $\left\{X_{i}\right\}$ be a sequence of independent random variables in a B-convex Banach space $\mathfrak{X}$ with $E\left(X_{i}\right)=0$, and $\operatorname{Var}\left(X_{i}\right) \leqslant 1, \forall i$. Then the sequence satisfies the SLLN.

4. Basic theorems in the new conditions.

6. THEOREM. Let $\left\{X_{\alpha} \mid \alpha \in A\right\}$ be a collection of random variables which have expectations and are independent. Then the collection is conditionally independent.

7. THEOREM. Let $\left\{X_{\alpha} \mid \alpha \in A\right\}$ be a collection of random variables which are independent and symmetric. Then the collection is mutually symmetric. 
8. THEOREM. Every collection of mutually symmetric random variables which have expectations is conditionally independent.

9. THEOREM. Every collection of conditionally independent random variables is weakly orthogonal.

5. The strong law of large numbers.

10. Theorem (Kolmogorov Strong LAW of LARge Numbers). Let $\left\{X_{i}\right\}$ be any conditionally independent sequence of random variables taking values in a Hilbert space, and satisfying the conditions that $E\left(X_{i}\right)=0, \forall i$, and $\sum_{i=1}^{\infty} \operatorname{Var}\left(X_{i}\right) / i^{2}<\infty$. Then the sequence $\left\{X_{i}\right\}$ satisfies the SLLN.

Proof. The conditional independence of the sequence $\left\{X_{i}\right\}$ guarantees that the sums $S_{n}=X_{1}+\cdots+X_{n}$ form a martingale. It is well known that the martingale condition is sufficient for the Kolmogorov inequality in real and complex spaces. A standard proof for the complex case (cf., e.g., Feller [7], mutatis mutandis) will prove the inequality for Hilbert space-valued random variables, and the theorem then follows from this inequality.

11. EXAmple (BeCK AND WARREN). Let the sequences $\left\{n_{i}\right\}$ and $\left\{p_{i}\right\}$ be chosen coverging to $\infty$, and let $\mathfrak{X}_{i}$ be the finite-dimensional Banach space of dimension $n_{i}$ with norm of order $p_{i}$. Define $\mathfrak{X}=\Sigma_{i=1}^{\infty} \bigoplus_{2} \mathfrak{X}_{i}$ to be the Hilbert space direct sum of the spaces $\mathfrak{X}_{i}$, i.e. the elements of $\mathfrak{X}$ are sequences $\left\{x_{i}\right\}$ with $x_{i} \in \mathfrak{X}_{i}$ and $\left\|\left\{x_{i}\right\}\right\|=\left(\Sigma_{i=1}^{\infty}\left\|x_{i}\right\|^{2}\right)^{1 / 2}$. Then $\mathfrak{X}$ is reflexive and separable, and if each $p_{i}>1$, it is locally uniformly convex as well.

We can construct in $\mathfrak{X}$ a sequence of random variables $X_{i}$ satisfying

(i) the sequence $\left\{X_{i}\right\}$ is mutually symmetric,

(ii) the $X_{i}$ are identically distributed,

(iii) the $X_{i}$ are uniformly bounded, i.e. $\left\|X_{i}(\omega)\right\| \leqslant 1, \forall i, \forall \omega$,

(iv) the SLLN fails for $\left\{X_{i}\right\}$.

Note that the sequence $\left\{X_{i}\right\}$ is, a fortiori, conditionally independent and weakly orthogonal.

12. Theorem. Let $\mathfrak{X}$ be a B-convex Banach space, and let $X_{i}$ be a mutually symmetric sequence of random $\mathfrak{X}$-variables satisfying $E\left(X_{i}\right)=0$ and $\left\|X_{i}(\omega)\right\| \leqslant 1, \forall i, \forall \omega$. Then $\left\{X_{i}\right\}$ satisfies the SLLN.

13. QuERY. It would be interesting to know whether Theorem 12 will still hold with the condition of mutual symmetry relaxed to conditional in- 
dependence, or even to weak orthogonality. I conjecture that the first of these is correct, but not the second. The process of "symmetrization" used for this purpose in Beck [3] will not go through verbatim, though a modification of the process (i.e. a symmetrization with respect to a measure in the direct product which is not the direct product of the measures) should suffice.

6. Conclusion. I believe that conditional independence is an important condition in probability theory, strong enough to produce some of the major results of finite-dimensional probability theory, but weak enough to be insufficient for others (such as the central limit theorem). In the area of the strong law of large numbers, the contrasts to be drawn among Theorems 2, 10, and 12, and Example 11 show that this condition, together with convexity condition (B), must live very close to the essential hypothesis for this theorem as a probabilistic entity.

\section{BIBLIOGRAPHY}

1. Anatole Beck, Une loi forte des grands nombres dans des espaces de Banach uniformément convexes, Ann. Inst. H. Poincaré 16 (1958), 35-45. MR 21 \#361.

2. - A convexity condition in Banach spaces and the strong law of large numbers, Proc. Amer. Math. Soc. 13 (1962), 329-334. MR 24 \#A3681.

3. - On the strong law of large numbers, Ergodic Theory (Proc. Internat. Sympos., Tulane Univ., New Orleans, La., 1961), Academic Press, New York, 1963, pp. 21-53. MR $28 \# 3470$.

4. Anatole Beck and Peter Warren, Weak orthogonality, Pacific J. Math. 41 (1972), 1-11. MR $46 \# 6006$.

5. - Strong laws of large numbers for weakly orthogonal sequences of Banach space-valued random variables, Ann. Probability (to appear).

6. - Counter examples to strong laws of large numbers for Banach spacevalued random variables, University of Denver preprints (to appear).

7. William Feller, An introduction to probability theory and its applications. Vol. II, Wiley, New York, 1966. MR 35 \#1048.

8. E. Mourier, Eléments aléatoires dans un espace de Banach, Ann. Inst. H. Poincaré 13 (1953), 161-244. MR 16, 268.

LONDON SCHOOL OF ECONOMICS, UNIVERSITY OF LONDON, LONDON, ENGLAND 